\title{
26. DATA REPORT: MAJOR, TRACE, AND RARE EARTH ELEMENT COMPOSITION OF INTERSTITIAL WATER SQUEEZE CAKES ${ }^{1}$
}

\author{
R.W. Murray, ${ }^{2}$ J.M. Gieskes, ${ }^{3}$ and R.C. Pflaum ${ }^{4}$
}

\section{INTRODUCTION}

On Ocean Drilling Program Leg 152 to the East Greenland Margin, interstitial water samples were taken on a regular basis to elucidate diagenetic chemical reactions occurring both within the sediment column and as a result of basement alteration. Although limited somewhat by poor recovery at Sites 914,915 , and 916 (i.e., the inshore sites), the shipboard interstitial water program managed to obtain more complete low-resolution profiles at Sites 918 and 919 (i.e., the more offshore sites). These results are detailed in Larson, Saunders, Clift, et al. (1994) and Gieskes et al. (this volume).

An important consideration during interpretation of the interstitial water results is the chemical composition of the solid phase enclosing those interstitial waters. To provide these data, we analyzed the squeeze cakes that resulted from interstitial water extrusion for a large suite of major, trace, and rare earth elements. These results, based on the analysis of 63 squeeze cakes, complement the more detailed sedimentary chemical profiles of Saito (this volume) and provide additional data on some elements not analyzed by Saito (this volume), such as the rare earth elements. Where appropriate, some of the data are used in the interpretations of Gieskes et al. (this volume). The data are provided here to facilitate future chemical studies of the sedimentary sequences sampled by Leg 152 and (as mentioned above) to assist in the interpretation of the interstitial water data.

\section{ANALYTICAL METHODS}

Samples were analyzed by inductively coupled plasma-emission spectrometry (ICP-ES) and inductively coupled plasma-mass spectrometry (ICP-MS). Sample preparation broadly followed the procedure described in Murray and Leinen (1993), but the procedure was slightly modified for the particular lithologies encountered during Leg 152, as described below. All acids used during the following sample preparation were double-distilled UltraPure grade from Seastar Chemicals (Seattle, WA, U.S.A.). Bulk sediment samples were freeze dried and subsequently hand powdered with an agate mortar and pestle. Complete digestion of $\sim 0.05 \mathrm{~g}$ of sample powder was achieved through a multistep protocol beginning with $\mathrm{HNO}_{3}$ and $\mathrm{HF}$ microwave-assisted dissolution in sealed Teflon vials $(15 \mathrm{~mL}$; Savillex Corp., MN, U.S.A.). Powder was poured directly into 0.5 $\mathrm{mL}$ of $\mathrm{HNO}_{3}$, allowed to react for $\sim 1 \mathrm{hr}$ and, following addition of 5 $\mathrm{mL}$ of concentrated HF, allowed to soak for at least $24 \mathrm{hr}$. The HF:silicate ratio was intentionally designed to be relatively high, thus assisting complete attack of terrigenous phases. Sealed Teflon vials were heated as a group in a commercial household microwave oven

'Saunders, A.D., Larsen, H.C., and Wise, S.W., Jr. (Eds.), 1998. Proc. ODP, Sci. Results, 152: College Station, TX (Ocean Drilling Program).

${ }^{2}$ Department of Earth Sciences, Boston University, Boston, MA 02215, U.S.A. rickm@bu.edu

${ }^{3}$ Scripps Institution of Oceanography, University of California, La Jolla, CA 92093, U.S.A.

${ }^{4}$ Earth and Planetary Science, Harvard University, Cambridge, MA 02138, U.S.A. (Present address: Department of Geology and Geophysics, University of Hawaii, 2525 Correa Road, Honolulu, HI 96822, U.S.A.) for $1.2 \mathrm{hr}$ at $10 \%$ power. This heating was followed by three hot-plate drydowns of additions of, in succession, $\mathrm{HNO}_{3}$, aqua regia, and $\mathrm{HNO}_{3}$. As a final step, $1.0 \mathrm{~mL}$ of $\mathrm{H}_{2} \mathrm{O}_{2}$ (Ultrex Ultrapure, Baker) was added to the final $1 \mathrm{~mL}$ of $\mathrm{HNO}_{3}$-based sample solution. All solutions were visually clear, with no residue apparent. For ICP-ES analysis, samples were diluted using trace-metal clean water to a 1:500 ratio (by mass) in precleaned, high-density Nalgene polyethylene bottles. Deviations from exact $500 \times$ dilution $(\leq 2 \%)$ were taken into account during data reduction. An $8-\mathrm{mL}$ aliquot was taken from this $500 \times$ dilution and diluted by a factor of 2 (by mass) to arrive at a second solution at $1000 \times$ dilution, for eventual use during ICP-MS analysis.

For the analysis of $\mathrm{P}, \mathrm{Mn}, \mathrm{Fe}, \mathrm{Al}, \mathrm{Ca}, \mathrm{Ti}, \mathrm{Sr}$, and $\mathrm{Ba}$, solutions were introduced by conventional nebulization into a Jobin-Yvon JY24 sequential ICP-ES and analyzed in comparison to matrixmatched synthetic standards. Standards were run at the beginning and end of each run. A drift-monitoring solution was run after every second standard and after every third sample. Blanks, internal references, and international Standard Reference Materials (SRM) were run in each batch as unknowns. Every solution, regardless of type, was analyzed in triplicate. Abundances of V (mass 51), Cr (52), Co (59), $\mathrm{Ni}$ (60), $\mathrm{Cu}$ (63 and 65), Zn (66 and 68), Y (89), Pb (206, 207, and 208), and the rare earth elements La (139), Ce (140), Pr (141), Nd (143, 145, and 146), Sm (147, 149, and 152), Eu (151 and 153), Gd (157 and 160), Tb (159), Dy (162 and 163), Ho (165), Er (166 and 168), Tm (169), Yb (171 and 174), and Lu (175) were determined by ICP-MS at Harvard University, using the isotopes indicated in parentheses. During ICP-MS analysis, an internal standard of $100 \mathrm{ppb}{ }^{115} \mathrm{In}$ was used to correct for ionization suppression, and the data were calibrated against synthetic standards bracketing the observed concentrations within the sample suite. For both ICP-ES and ICP-MS analysis, samples were weighed, dissolved, and run in different random orders at each stage of preparation (i.e., completely mixing site numbers and depths).

Precision was estimated by complete quadruplicate analysis (i.e., from the powder weighing step onward) of Sample 152-918A-10H$4,145-150 \mathrm{~cm}$. For the elements determined by ICP-ES, precision is always better than $3 \%$ (conservative), except for $\mathrm{Ba}$, which is within $10 \%$. For the elements determined by ICP-MS, precision is $\sim 5 \%$ for $\mathrm{V}, \mathrm{Ni}, \mathrm{Cu}, \mathrm{Y}$, and $\mathrm{Ce} ; \sim 10 \%$ for $\mathrm{Zn}, \mathrm{La}, \mathrm{Pr}, \mathrm{Gd}, \mathrm{Tb}$, and $\mathrm{Ho} ; \sim 15 \%$ for $\mathrm{Cr}, \mathrm{Co}, \mathrm{Nd}, \mathrm{Sm}, \mathrm{Eu}, \mathrm{Dy}, \mathrm{Er}$, and $\mathrm{Pb}$; and between $15 \%$ and $20 \%$ for $\mathrm{Tm}, \mathrm{Yb}$, and $\mathrm{Lu}$. Unfortunately, not all samples were analyzed for $\mathrm{Ni}$ and $\mathrm{Cu}$ because of machine difficulties. Accuracy was more difficult to assess. Standard Reference Material BCSS-1 (an estuarine sediment from the Gulf of St. Lawrence, available from the National Research Council of Canada) was analyzed with the samples. Results of this analysis were consistently 5\%-10\% lower than the accepted (yet poorly constrained) values for many of the more refractory elements, although elemental ratios show good agreement. We noted, however, that our preparation scheme did not completely digest BCSS-1, as evidenced by the presence of several ( 10) dark grains at the end of the dissolution protocol. Such incomplete dissolution was not observed in the preparation of the Leg 152 samples themselves. We therefore estimate that accuracy is within precision. In fact, these contrasts in dissolution are consistent with the different lithology of the Gulf of St. Lawrence SRM and the open ocean Leg 152 samples. 


\section{RESULTS}

Analytical results for ICP-ES analysis are given in Table 1, and the results for ICP-MS analysis are given in Table 2.

\section{ACKNOWLEDGMENTS}

We thank the extremely helpful technical staff of the Leg 152 ODP shipboard personnel. John Brader helped in sample preparation at Boston University. Reviews provided by S.M. McLennan and R.M. Owen are appreciated. Research was funded by JOI/USSAC Cruise Science Support grants to R.W. Murray and J. Gieskes.

\section{REFERENCES}

Larsen, H.C., Saunders, A.D., Clift, P.D., et al., 1994. Proc. ODP, Init. Repts., 152: College Station, TX (Ocean Drilling Program).

Murray, R.W., and Leinen, M., 1993. Chemical transport to the seafloor of the equatorial Pacific Ocean across a latitudinal transect at $135^{\circ} \mathrm{W}$ : tracking sedimentary major, trace, and rare earth element fluxes at the Equator and the Intertropical Convergence Zone. Geochim. Cosmochim. Acta., $57: 4141-4163$

Date of initial receipt: 1 November 1995

Date of acceptance: 22 May 1996

Ms 152SR-229 
Table 1. ICP-ES results, bulk sedimentary chemistry of interstitial water squeeze cakes, Leg 152.

\begin{tabular}{|c|c|c|c|c|c|c|c|c|c|}
\hline $\begin{array}{l}\text { Core, section, } \\
\text { interval }(\mathrm{cm})\end{array}$ & $\begin{array}{l}\text { Depth } \\
\text { (mbsf) }\end{array}$ & $\begin{array}{c}\mathrm{P} \\
(\mathrm{ppm})\end{array}$ & $\begin{array}{c}\mathrm{Mn} \\
(\mathrm{ppm})\end{array}$ & $\begin{array}{l}\mathrm{Fe} \\
(\%)\end{array}$ & $\begin{array}{c}\mathrm{Al} \\
(\%)\end{array}$ & $\begin{array}{l}\mathrm{Ca} \\
(\%)\end{array}$ & $\begin{array}{c}\mathrm{Ti} \\
(\%)\end{array}$ & $\begin{array}{c}\mathrm{Sr} \\
(\mathrm{ppm})\end{array}$ & $\begin{array}{c}\mathrm{Ba} \\
(\mathrm{ppm})\end{array}$ \\
\hline $\begin{array}{l}152-914 \mathrm{~A}- \\
1 \mathrm{H}-2,140-150\end{array}$ & 2.90 & 580 & 770 & 4.68 & 6.98 & 3.26 & 0.487 & 260 & 500 \\
\hline $\begin{array}{l}\text { 152-914B- } \\
\text { 15R-1, 69-77 } \\
\text { 17R-4, 140-150 }\end{array}$ & $\begin{array}{l}216.79 \\
241.40\end{array}$ & $\begin{array}{l}520 \\
470\end{array}$ & $\begin{array}{l}720 \\
580\end{array}$ & $\begin{array}{l}6.94 \\
6.97\end{array}$ & $\begin{array}{l}6.67 \\
6.36\end{array}$ & $\begin{array}{l}2.41 \\
1.90\end{array}$ & $\begin{array}{l}1.19 \\
10.1\end{array}$ & $\begin{array}{l}330 \\
200\end{array}$ & $\begin{array}{l}450 \\
340\end{array}$ \\
\hline $\begin{array}{l}\text { 152-915A- } \\
\text { 1R-1, 145-150 } \\
\text { 15R-1, 0-5 } \\
\text { 18R-2, 140-150 } \\
\text { 19R-2, 140-150 } \\
\text { 21R-5, 46-56 } \\
\text { 22R-2, 145-150 }\end{array}$ & $\begin{array}{r}1.45 \\
121.20 \\
151.50 \\
161.00 \\
173.30 \\
180.45\end{array}$ & $\begin{array}{r}520 \\
2450 \\
1900 \\
870 \\
480 \\
460\end{array}$ & $\begin{array}{r}730 \\
1950 \\
6450 \\
630 \\
600 \\
650\end{array}$ & $\begin{array}{c}4.70 \\
8.13 \\
7.06 \\
14.7 \\
9.15 \\
9.23\end{array}$ & $\begin{array}{c}6.78 \\
3.93 \\
6.19 \\
12.6 \\
8.51 \\
8.67\end{array}$ & $\begin{array}{l}2.90 \\
8.18 \\
19.4 \\
0.095 \\
1.07 \\
0.709\end{array}$ & $\begin{array}{l}0.453 \\
0.362 \\
1.01 \\
2.12 \\
1.26 \\
1.27\end{array}$ & $\begin{array}{r}140 \\
260 \\
20 \\
\text { bdl } \\
250 \\
75\end{array}$ & $\begin{array}{r}440 \\
300 \\
90 \\
130 \\
370 \\
280\end{array}$ \\
\hline $\begin{array}{l}152-916 \mathrm{~A}- \\
13 \mathrm{R}-2,140-150\end{array}$ & 81.50 & 1910 & 1450 & 10.6 & 9.39 & 1.05 & 1.08 & 80 & 160 \\
\hline $\begin{array}{l}152-918 \mathrm{~B}- \\
1 \mathrm{H}-3,145-150\end{array}$ & 4.45 & 720 & 810 & 5.09 & 6.54 & 3.73 & 0.722 & 250 & 410 \\
\hline $\begin{array}{l}152-918 \mathrm{~A}- \\
2 \mathrm{H}-4,150-155\end{array}$ & 7.75 & 810 & 820 & 5.44 & 7.48 & 3.28 & 0.717 & 275 & 520 \\
\hline $\begin{array}{l}152-918 \mathrm{~B}- \\
2 \mathrm{H}-4,145-150 \\
3 \mathrm{H}-5,145-150\end{array}$ & $\begin{array}{l}12.75 \\
23.75\end{array}$ & $\begin{array}{l}770 \\
770\end{array}$ & $\begin{array}{l}690 \\
790\end{array}$ & $\begin{array}{l}3.93 \\
5.40\end{array}$ & $\begin{array}{l}6.99 \\
7.08\end{array}$ & $\begin{array}{l}3.86 \\
3.31\end{array}$ & $\begin{array}{l}0.465 \\
0.644\end{array}$ & $\begin{array}{l}380 \\
280\end{array}$ & $\begin{array}{l}570 \\
490\end{array}$ \\
\hline $\begin{array}{l}152-918 \mathrm{~A}- \\
4 \mathrm{H}-4,145-150\end{array}$ & 26.75 & 790 & 730 & 5.32 & 7.73 & 2.66 & 0.556 & 300 & 750 \\
\hline $\begin{array}{l}152-918 \mathrm{C}- \\
1 \mathrm{H}-4,145-150\end{array}$ & 31.75 & 660 & 950 & 5.83 & 5.74 & 6.22 & 0.915 & 300 & 290 \\
\hline $\begin{array}{l}152-918 \mathrm{~A}- \\
7 \mathrm{H}-4,145-150 \\
10 \mathrm{H}-4,145-150 \\
13 \mathrm{H}-4,145-150 \\
16 \mathrm{H}-4,140-150 \\
19 \mathrm{H}-4,140-150 \\
23 \mathrm{X}-4,140-150 \\
26 \mathrm{X}-4,140-150 \\
31 \mathrm{X}-4,0-10 \\
37 \mathrm{X}-5,90-100\end{array}$ & $\begin{array}{r}55.25 \\
83.75 \\
112.25 \\
139.20 \\
167.70 \\
205.70 \\
232.40 \\
275.10 \\
322.00\end{array}$ & $\begin{array}{r}780 \\
850 \\
760 \\
1010 \\
850 \\
750 \\
920 \\
880 \\
840\end{array}$ & $\begin{array}{r}650 \\
1430 \\
650 \\
1190 \\
1190 \\
910 \\
1110 \\
630 \\
1000\end{array}$ & $\begin{array}{l}4.63 \\
7.66 \\
6.03 \\
8.37 \\
7.76 \\
6.19 \\
8.24 \\
5.31 \\
6.99\end{array}$ & $\begin{array}{l}6.88 \\
6.35 \\
7.56 \\
6.80 \\
6.98 \\
6.09 \\
6.87 \\
7.67 \\
6.62\end{array}$ & $\begin{array}{l}3.10 \\
6.22 \\
2.47 \\
5.68 \\
5.32 \\
3.91 \\
4.57 \\
2.51 \\
4.36\end{array}$ & $\begin{array}{l}0.534 \\
1.22 \\
0.619 \\
1.25 \\
1.13 \\
0.990 \\
1.20 \\
0.574 \\
1.10\end{array}$ & $\begin{array}{l}290 \\
290 \\
240 \\
310 \\
170 \\
170 \\
150 \\
280 \\
320\end{array}$ & $\begin{array}{l}620 \\
230 \\
650 \\
250 \\
210 \\
350 \\
210 \\
650 \\
330\end{array}$ \\
\hline $\begin{array}{l}\text { 152-918D- } \\
\text { 13R-1, 0-5 } \\
\text { 22R-2, 140-150 } \\
\text { 25R-3, 149-157 } \\
\text { 28R-3, 140-150 } \\
\text { 31R-CC, 5-8 } \\
\text { 34R-1, 2-10 } \\
\text { 37R-1, 140-150 } \\
\text { 40R-1, 140-150 } \\
\text { 44R-2, 139-150 } \\
\text { 47R-1, 50-60 } \\
\text { 51R-2, 140-150 } \\
\text { 55R-4, 112-122 } \\
\text { 62R-1, 121-129 } \\
\text { 68R-1, 91-100 } \\
\text { 74R-1, 3-14 }\end{array}$ & $\begin{array}{l}403.90 \\
486.70 \\
515.86 \\
546.20 \\
571.75 \\
599.62 \\
630.00 \\
658.90 \\
698.99 \\
725.10 \\
766.10 \\
807.42 \\
870.51 \\
925.91 \\
982.73\end{array}$ & $\begin{array}{l}700 \\
560 \\
560 \\
650 \\
420 \\
400 \\
480 \\
470 \\
480 \\
470 \\
470 \\
400 \\
360 \\
960\end{array}$ & $\begin{array}{l}630 \\
890 \\
750 \\
750 \\
600 \\
550 \\
480 \\
380 \\
440 \\
460 \\
400 \\
310 \\
450 \\
440\end{array}$ & $\begin{array}{l}4.67 \\
6.51 \\
4.52 \\
6.45 \\
7.26 \\
4.92 \\
6.27 \\
5.34 \\
5.00 \\
6.75 \\
5.15 \\
5.65 \\
4.94 \\
7.59\end{array}$ & $\begin{array}{l}7.11 \\
6.66 \\
5.78 \\
7.82 \\
6.91 \\
5.38 \\
7.08 \\
5.88 \\
5.39 \\
6.19 \\
5.84 \\
5.79 \\
4.82 \\
5.42\end{array}$ & $\begin{array}{l}2.96 \\
3.74 \\
5.06 \\
2.43 \\
1.75 \\
1.73 \\
4.83 \\
6.02 \\
9.75 \\
3.71 \\
8.50 \\
5.15 \\
5.16 \\
1.61\end{array}$ & $\begin{array}{l}0.550 \\
1.05 \\
0.686 \\
1.19 \\
1.04 \\
0.866 \\
0.847 \\
0.708 \\
0.699 \\
0.983 \\
0.800 \\
0.770 \\
0.724 \\
0.976\end{array}$ & $\begin{array}{l}300 \\
200 \\
180 \\
180 \\
120 \\
120 \\
340 \\
340 \\
470 \\
190 \\
480 \\
370 \\
290 \\
120\end{array}$ & $\begin{array}{l}590 \\
340 \\
410 \\
360 \\
360 \\
400 \\
360 \\
450 \\
450 \\
630 \\
710 \\
630 \\
360 \\
450\end{array}$ \\
\hline $\begin{array}{l}80 \mathrm{R}-1,0-11 \\
83 \mathrm{R}-1,136-141 \\
88 \mathrm{R}-1,93-100 \\
91 \mathrm{R}-1,119-129 \\
95 \mathrm{R}-2,140-150\end{array}$ & $\begin{array}{l}1040.60 \\
1070.96 \\
1118.73 \\
1147.69 \\
1182.20\end{array}$ & $\begin{array}{r}1040 \\
400 \\
540 \\
1110 \\
100\end{array}$ & $\begin{array}{r}860 \\
950 \\
1990 \\
350 \\
310\end{array}$ & $\begin{array}{c}4.38 \\
4.10 \\
6.87 \\
6.18 \\
10.7\end{array}$ & $\begin{array}{l}5.10 \\
4.82 \\
3.87 \\
6.40 \\
4.30\end{array}$ & $\begin{array}{c}4.16 \\
3.84 \\
16.9 \\
5.04 \\
0.385\end{array}$ & $\begin{array}{l}0.693 \\
0.555 \\
0.844 \\
0.812 \\
0.480\end{array}$ & $\begin{array}{r}140 \\
130 \\
560 \\
400 \\
40\end{array}$ & $\begin{array}{r}420 \\
470 \\
320 \\
2700 \\
190\end{array}$ \\
\hline $\begin{array}{l}152-919 \mathrm{~A}- \\
1 \mathrm{H}-2,145-150 \\
1 \mathrm{H}-4,145-150 \\
2 \mathrm{H}-2,145-150 \\
2 \mathrm{H}-4,145-150 \\
3 \mathrm{H}-2,145-150 \\
3 \mathrm{H}-4,145-150 \\
4 \mathrm{H}-2,145-150 \\
4 \mathrm{H}-4,145-150 \\
5 \mathrm{H}-2,145-150 \\
5 \mathrm{H}-4,145-150 \\
6 \mathrm{H}-3,145-150 \\
7 \mathrm{H}-3,145-150 \\
8 \mathrm{H}-3,145-150 \\
9 \mathrm{H}-3,145-150 \\
10 \mathrm{H}-3,145-150\end{array}$ & $\begin{array}{r}2.95 \\
5.95 \\
10.95 \\
13.95 \\
20.45 \\
23.45 \\
29.95 \\
32.95 \\
39.45 \\
42.45 \\
50.45 \\
59.95 \\
69.45 \\
78.95 \\
88.45\end{array}$ & $\begin{array}{r}840 \\
850 \\
730 \\
780 \\
800 \\
800 \\
900 \\
750 \\
830 \\
790 \\
710 \\
580 \\
1200 \\
850 \\
730\end{array}$ & $\begin{array}{r}1160 \\
840 \\
990 \\
980 \\
960 \\
1120 \\
1100 \\
830 \\
1260 \\
1220 \\
1140 \\
540 \\
1110 \\
740 \\
1150\end{array}$ & $\begin{array}{l}7.02 \\
6.11 \\
6.48 \\
6.44 \\
5.96 \\
6.83 \\
7.57 \\
6.20 \\
7.13 \\
8.00 \\
7.15 \\
4.33 \\
6.74 \\
5.82 \\
6.81\end{array}$ & $\begin{array}{l}6.64 \\
7.62 \\
6.03 \\
5.99 \\
7.12 \\
6.16 \\
6.75 \\
6.76 \\
6.73 \\
6.57 \\
6.05 \\
7.11 \\
6.56 \\
6.61 \\
6.61\end{array}$ & $\begin{array}{l}5.97 \\
3.07 \\
4.67 \\
6.00 \\
4.72 \\
5.58 \\
4.84 \\
2.98 \\
6.46 \\
5.98 \\
6.53 \\
2.27 \\
5.18 \\
3.38 \\
6.17\end{array}$ & $\begin{array}{l}1.07 \\
0.644 \\
0.925 \\
0.950 \\
0.840 \\
1.04 \\
0.991 \\
0.800 \\
1.07 \\
1.19 \\
1.08 \\
0.458 \\
1.10 \\
0.813 \\
1.08\end{array}$ & $\begin{array}{l}390 \\
260 \\
190 \\
220 \\
270 \\
200 \\
180 \\
140 \\
240 \\
190 \\
240 \\
120 \\
350 \\
160 \\
210\end{array}$ & $\begin{array}{l}330 \\
620 \\
300 \\
260 \\
440 \\
240 \\
250 \\
380 \\
210 \\
200 \\
220 \\
440 \\
360 \\
310 \\
260\end{array}$ \\
\hline $\begin{array}{l}\text { 152-919B- } \\
3 \mathrm{H}-3,145-150 \\
4 \mathrm{H}-3,145-150 \\
7 \mathrm{H}-3,145-150\end{array}$ & $\begin{array}{r}94.45 \\
103.95 \\
132.45\end{array}$ & $\begin{array}{l}700 \\
780 \\
900\end{array}$ & $\begin{array}{l}890 \\
900 \\
720\end{array}$ & $\begin{array}{l}6.90 \\
6.47 \\
6.06\end{array}$ & $\begin{array}{l}6.45 \\
6.99 \\
7.69\end{array}$ & $\begin{array}{l}3.74 \\
4.16 \\
2.62\end{array}$ & $\begin{array}{l}0.927 \\
0.890 \\
0.623\end{array}$ & $\begin{array}{l}160 \\
170 \\
260\end{array}$ & $\begin{array}{l}320 \\
290 \\
700\end{array}$ \\
\hline
\end{tabular}




\begin{tabular}{|c|c|c|c|c|c|c|c|c|c|c|c|c|c|c|c|c|c|c|c|c|c|c|c|}
\hline $\begin{array}{l}\text { Core, section, } \\
\text { interval }(\mathrm{cm})\end{array}$ & $\begin{array}{l}\text { Depth } \\
\text { (mbsf) }\end{array}$ & $\begin{array}{c}\mathrm{V} \\
(\mathrm{ppm})\end{array}$ & $\begin{array}{c}\mathrm{Cr} \\
(\mathrm{ppm})\end{array}$ & $\begin{array}{c}\mathrm{Co} \\
(\mathrm{ppm})\end{array}$ & $\begin{array}{c}\mathrm{Ni} \\
(\mathrm{ppm})\end{array}$ & $\begin{array}{c}\mathrm{Cu} \\
(\mathrm{ppm})\end{array}$ & $\begin{array}{c}\mathrm{Zn} \\
(\mathrm{ppm})\end{array}$ & $\begin{array}{c}\mathrm{Y} \\
(\mathrm{ppm})\end{array}$ & $\begin{array}{c}\mathrm{La} \\
(\mathrm{ppm})\end{array}$ & $\begin{array}{c}\mathrm{Ce} \\
(\mathrm{ppm})\end{array}$ & $\begin{array}{c}\mathrm{Pr} \\
(\mathrm{ppm})\end{array}$ & $\begin{array}{c}\mathrm{Nd} \\
(\mathrm{ppm})\end{array}$ & $\begin{array}{l}\mathrm{Sm} \\
(\mathrm{ppm})\end{array}$ & $\begin{array}{c}\mathrm{Eu} \\
(\mathrm{ppm})\end{array}$ & $\begin{array}{c}\mathrm{Gd} \\
(\mathrm{ppm})\end{array}$ & $\begin{array}{c}\mathrm{Tb} \\
(\mathrm{ppm})\end{array}$ & $\begin{array}{c}\text { Dy } \\
(\mathrm{ppm})\end{array}$ & $\begin{array}{l}\text { Ho } \\
(\mathrm{ppm})\end{array}$ & $\begin{array}{c}\text { Er } \\
(\mathrm{ppm})\end{array}$ & $\begin{array}{c}\mathrm{Tm} \\
(\mathrm{ppm})\end{array}$ & $\begin{array}{c}\mathrm{Yb} \\
(\mathrm{ppm})\end{array}$ & $\begin{array}{c}\mathrm{Lu} \\
(\mathrm{ppm})\end{array}$ & $\begin{array}{c}\mathrm{Pb} \\
(\mathrm{ppm})\end{array}$ \\
\hline $\begin{array}{l}152-914 \mathrm{~A}- \\
1 \mathrm{H}-2,140-150\end{array}$ & 2.90 & 158 & 122 & 27 & 75 & 140 & 104 & 13.3 & 22.3 & 42.9 & 5.27 & 19.6 & 3.57 & 0.92 & 3.14 & 0.38 & 2.55 & 0.42 & 1.22 & 0.18 & 1.21 & 0.19 & 7.57 \\
\hline $\begin{array}{l}\text { 152-914B- } \\
\text { 15R-1, 69-77 } \\
17 \mathrm{R}-4,140-150\end{array}$ & $\begin{array}{l}216.79 \\
241.40\end{array}$ & $\begin{array}{l}280 \\
171\end{array}$ & $\begin{array}{l}319 \\
189\end{array}$ & $\begin{array}{l}44 \\
26\end{array}$ & 124 & 111 & $\begin{array}{l}166 \\
122\end{array}$ & $\begin{array}{l}20.7 \\
18.3\end{array}$ & $\begin{array}{l}38.0 \\
46.9\end{array}$ & $\begin{array}{l}72.8 \\
87.5\end{array}$ & $\begin{array}{r}8.23 \\
11.02\end{array}$ & $\begin{array}{l}29.5 \\
41.8\end{array}$ & $\begin{array}{l}5.52 \\
7.47\end{array}$ & $\begin{array}{l}1.28 \\
1.74\end{array}$ & $\begin{array}{l}4.80 \\
5.72\end{array}$ & $\begin{array}{l}0.64 \\
0.93\end{array}$ & $\begin{array}{l}3.93 \\
5.50\end{array}$ & $\begin{array}{l}0.60 \\
0.80\end{array}$ & $\begin{array}{l}1.85 \\
2.41\end{array}$ & $\begin{array}{l}0.22 \\
0.44\end{array}$ & $\begin{array}{l}1.48 \\
2.73\end{array}$ & $\begin{array}{l}0.24 \\
0.37\end{array}$ & $\begin{array}{l}4.77 \\
7.06\end{array}$ \\
\hline $\begin{array}{l}\text { 152-915A- } \\
\text { 1R-1, 145-150 } \\
\text { 15R-1, 0-5 } \\
\text { 18R-2, 140-150 } \\
\text { 19R-2, 140-150 } \\
\text { 21R-5, 46-56 } \\
\text { 22R-2, 145-150 }\end{array}$ & $\begin{array}{r}1.45 \\
121.20 \\
151.50 \\
161.00 \\
173.30 \\
180.45\end{array}$ & $\begin{array}{l}142 \\
242 \\
192 \\
398 \\
286 \\
318\end{array}$ & $\begin{array}{l}113 \\
148 \\
133 \\
364 \\
292 \\
424\end{array}$ & $\begin{array}{l}25 \\
37 \\
30 \\
77 \\
60 \\
51\end{array}$ & 202 & 211 & $\begin{array}{r}96 \\
107 \\
68 \\
153 \\
162 \\
595\end{array}$ & $\begin{array}{l}12.2 \\
29.1 \\
43.2 \\
18.0 \\
18.0 \\
17.5\end{array}$ & $\begin{array}{l}22.6 \\
24.4 \\
31.2 \\
21.7 \\
21.8 \\
24.7\end{array}$ & $\begin{array}{l}42.8 \\
56.2 \\
79.7 \\
50.6 \\
47.7 \\
53.7\end{array}$ & $\begin{array}{l}5.63 \\
5.64 \\
9.33 \\
6.53 \\
5.44 \\
6.44\end{array}$ & $\begin{array}{l}21.3 \\
21.3 \\
42.1 \\
27.8 \\
20.5 \\
28.1\end{array}$ & $\begin{array}{l}3.73 \\
4.21 \\
9.30 \\
5.65 \\
4.09 \\
5.55\end{array}$ & $\begin{array}{l}1.07 \\
1.17 \\
2.84 \\
1.83 \\
1.16 \\
1.61\end{array}$ & $\begin{array}{r}3.41 \\
4.59 \\
10.30 \\
5.81 \\
3.58 \\
5.38\end{array}$ & $\begin{array}{l}0.43 \\
0.69 \\
1.52 \\
0.85 \\
0.52 \\
0.75\end{array}$ & $\begin{array}{r}2.76 \\
4.12 \\
10.45 \\
5.84 \\
3.15 \\
4.87\end{array}$ & $\begin{array}{l}0.42 \\
0.65 \\
1.77 \\
0.93 \\
0.46 \\
0.82\end{array}$ & $\begin{array}{l}1.36 \\
2.06 \\
5.69 \\
2.83 \\
1.38 \\
2.29\end{array}$ & $\begin{array}{l}0.22 \\
0.26 \\
0.83 \\
0.41 \\
0.19 \\
0.38\end{array}$ & $\begin{array}{l}1.20 \\
1.65 \\
5.24 \\
2.84 \\
1.35 \\
2.30\end{array}$ & $\begin{array}{l}0.20 \\
0.26 \\
0.91 \\
0.56 \\
0.23 \\
0.36\end{array}$ & $\begin{array}{l}8.55 \\
2.71 \\
4.07 \\
7.00 \\
4.88 \\
7.71\end{array}$ \\
\hline $\begin{array}{l}152-916 \mathrm{~A}- \\
13 \mathrm{R}-2,140-150\end{array}$ & 81.50 & 293 & 311 & 67 & & & 135 & 29.0 & 17.4 & 32.2 & 4.78 & 20.9 & 4.70 & 1.51 & 4.92 & 0.76 & 5.34 & 0.91 & 2.82 & 0.39 & 2.70 & 0.44 & 3.44 \\
\hline $\begin{array}{l}\text { 152-918B- } \\
1 \mathrm{H}-3,145-150\end{array}$ & 4.45 & 183 & 126 & 23 & & & 84 & 19.5 & 26.3 & 50.0 & 6.81 & 28.0 & 5.19 & 1.44 & 4.73 & 0.63 & 4.02 & 0.67 & 2.07 & 0.30 & 1.91 & 0.27 & 8.52 \\
\hline $\begin{array}{l}\text { 152-918A- } \\
2 \mathrm{H}-4,150-155\end{array}$ & 7.75 & 150 & 98 & 24 & & & 98 & 18.0 & 35.8 & 65.6 & 8.54 & 35.5 & 6.21 & 1.65 & 5.64 & 0.77 & 5.06 & 0.80 & 2.44 & 0.34 & 2.42 & 0.37 & 11.53 \\
\hline $\begin{array}{l}\text { 152-918B- } \\
2 \mathrm{H}-4,145-150 \\
3 \mathrm{H}-5,145-150\end{array}$ & $\begin{array}{l}12.75 \\
23.75\end{array}$ & $\begin{array}{l}123 \\
150\end{array}$ & $\begin{array}{l}97 \\
96\end{array}$ & $\begin{array}{l}18 \\
23\end{array}$ & & & $\begin{array}{l}70 \\
95\end{array}$ & $\begin{array}{l}16.3 \\
17.5\end{array}$ & $\begin{array}{l}27.4 \\
30.9\end{array}$ & $\begin{array}{l}51.1 \\
57.4\end{array}$ & $\begin{array}{l}6.78 \\
7.49\end{array}$ & $\begin{array}{l}26.8 \\
30.8\end{array}$ & $\begin{array}{l}4.73 \\
5.42\end{array}$ & $\begin{array}{l}1.29 \\
1.50\end{array}$ & $\begin{array}{l}3.94 \\
4.57\end{array}$ & $\begin{array}{l}0.55 \\
0.77\end{array}$ & $\begin{array}{l}3.41 \\
4.25\end{array}$ & $\begin{array}{l}0.62 \\
0.67\end{array}$ & $\begin{array}{l}1.73 \\
2.04\end{array}$ & $\begin{array}{l}0.23 \\
0.33\end{array}$ & $\begin{array}{l}1.93 \\
1.88\end{array}$ & $\begin{array}{l}0.25 \\
0.33\end{array}$ & $\begin{array}{l}8.73 \\
9.33\end{array}$ \\
\hline $\begin{array}{l}152-918 \mathrm{~A}- \\
4 \mathrm{H}-4,145-150\end{array}$ & 26.75 & 130 & 113 & 25 & & & 116 & 15.4 & 42.1 & 76.0 & 9.88 & 37.1 & 6.28 & 1.57 & 5.04 & 0.69 & 3.81 & 0.61 & 1.92 & 0.32 & 1.77 & 0.28 & 11.61 \\
\hline $\begin{array}{l}152-918 \mathrm{C}- \\
1 \mathrm{H}-4,145-150\end{array}$ & 31.75 & 164 & 130 & 27 & & & 93 & 21.9 & 19.8 & 42.7 & 5.05 & 22.5 & 4.81 & 1.51 & 5.00 & 0.79 & 4.68 & 0.84 & 2.52 & 0.34 & 1.93 & 0.33 & 5.42 \\
\hline $\begin{array}{c}152-918 \mathrm{~A}- \\
7 \mathrm{H}-4,145-150 \\
10 \mathrm{H}-4,145-150 \\
13 \mathrm{H}-4,145-150 \\
16 \mathrm{H}-4,140-150 \\
19 \mathrm{H}-4,140-150 \\
23 \mathrm{X}-4,140-150 \\
26 \mathrm{X}-4,140-150 \\
31 \mathrm{X}-4,0-10 \\
37 \mathrm{X}-5,90-100\end{array}$ & $\begin{array}{r}55.25 \\
83.75 \\
112.25 \\
139.20 \\
167.70 \\
205.70 \\
232.40 \\
275.10 \\
322.00\end{array}$ & $\begin{array}{l}149 \\
299 \\
131 \\
298 \\
273 \\
192 \\
277 \\
126 \\
271\end{array}$ & $\begin{array}{r}101 \\
141 \\
93 \\
131 \\
114 \\
238 \\
107 \\
97 \\
215\end{array}$ & $\begin{array}{l}21 \\
47 \\
22 \\
49 \\
40 \\
46 \\
38 \\
20 \\
44\end{array}$ & $\begin{array}{l}82 \\
76\end{array}$ & $\begin{array}{l}162 \\
164\end{array}$ & $\begin{array}{r}95 \\
139 \\
115 \\
134 \\
115 \\
97 \\
125 \\
110 \\
132\end{array}$ & $\begin{array}{l}17.1 \\
30 \\
16.4 \\
26.5 \\
25.8 \\
22.6 \\
25.9 \\
15.6 \\
25.8\end{array}$ & $\begin{array}{l}29.7 \\
20 \\
39.2 \\
14.9 \\
19.4 \\
27.9 \\
21.6 \\
39.5 \\
20.2\end{array}$ & $\begin{array}{l}53.3 \\
42 \\
70.6 \\
32.8 \\
38.2 \\
51.0 \\
43.4 \\
70.7 \\
42.9\end{array}$ & $\begin{array}{l}6.96 \\
5.67 \\
8.87 \\
4.38 \\
5.45 \\
7.04 \\
6.39 \\
9.36 \\
5.14\end{array}$ & $\begin{array}{l}28.4 \\
22.59 \\
35.2 \\
18.2 \\
24.7 \\
29.9 \\
27.5 \\
34.7 \\
21.0\end{array}$ & $\begin{array}{l}4.85 \\
5.03 \\
5.80 \\
4.33 \\
5.36 \\
5.97 \\
5.84 \\
6.05 \\
4.64\end{array}$ & $\begin{array}{l}1.33 \\
1.51 \\
1.66 \\
1.38 \\
1.78 \\
1.70 \\
1.75 \\
1.52 \\
1.31\end{array}$ & $\begin{array}{l}4.27 \\
5.49 \\
5.43 \\
4.41 \\
5.83 \\
5.57 \\
6.46 \\
5.06 \\
4.41\end{array}$ & $\begin{array}{l}0.57 \\
0.84 \\
0.71 \\
0.65 \\
0.88 \\
0.84 \\
0.97 \\
0.75 \\
0.63\end{array}$ & $\begin{array}{l}3.43 \\
5.40 \\
4.48 \\
4.49 \\
5.87 \\
6.01 \\
6.47 \\
4.37 \\
3.69\end{array}$ & $\begin{array}{l}0.58 \\
0.89 \\
0.77 \\
0.79 \\
0.97 \\
0.95 \\
1.06 \\
0.68 \\
0.63\end{array}$ & $\begin{array}{l}1.87 \\
2.80 \\
2.46 \\
2.27 \\
3.08 \\
3.11 \\
3.32 \\
1.99 \\
1.76\end{array}$ & $\begin{array}{l}0.26 \\
0.39 \\
0.37 \\
0.29 \\
0.42 \\
0.48 \\
0.43 \\
0.27 \\
0.24\end{array}$ & $\begin{array}{l}1.74 \\
2.49 \\
2.06 \\
1.96 \\
2.98 \\
3.18 \\
2.75 \\
2.03 \\
1.62\end{array}$ & $\begin{array}{l}0.24 \\
0.41 \\
0.30 \\
0.31 \\
0.41 \\
0.45 \\
0.43 \\
0.28 \\
0.26\end{array}$ & $\begin{array}{r}8.94 \\
2.73 \\
12.28 \\
2.98 \\
4.83 \\
7.00 \\
5.48 \\
12.46 \\
4.50\end{array}$ \\
\hline $152-918$ & & & & & & 170 & 152 & 25.8 & 20.2 & 42.9 & 5.14 & 21.0 & 4.64 & 1.31 & 4.41 & 0.63 & 3.69 & 0.63 & 1.76 & 0.24 & 1.62 & 0.26 & 4.50 \\
\hline & & & & & & & & & & & & & & & & & & & & & & & \\
\hline & 403.9 & 121 & 97 & 22 & & & 91 & 1 & 32 & 57.4 & 7.30 & 30.1 & 4.71 & 1.44 & 4.49 & & 3.68 & & 1. & & 1. & 0 & 10.73 \\
\hline 25 & $\begin{array}{l}\begin{array}{l}486.7 \\
515.8\end{array} \\
515\end{array}$ & $\begin{array}{l}210 \\
130\end{array}$ & $\begin{array}{r}136 \\
72\end{array}$ & $\begin{array}{l}27 \\
18\end{array}$ & & & $\begin{array}{l}92 \\
75\end{array}$ & $\begin{array}{l}20.2 \\
15.6\end{array}$ & $\begin{array}{l}23.0 \\
23.2\end{array}$ & $\begin{array}{l}42.9 \\
44.4\end{array}$ & $\begin{array}{l}6.01 \\
6.03\end{array}$ & $\begin{array}{l}25.2 \\
24.2\end{array}$ & $\begin{array}{l}5.16 \\
4.48\end{array}$ & $\begin{array}{l}1.11 \\
1.13\end{array}$ & $\begin{array}{l}5.16 \\
4.20\end{array}$ & $\begin{array}{l}0.74 \\
0.60\end{array}$ & $\begin{array}{l}5.01 \\
4.12\end{array}$ & $\begin{array}{l}0.8 \\
0.6\end{array}$ & $\begin{array}{l}2.23 \\
2.01\end{array}$ & $\begin{array}{l}0.33 \\
0.34\end{array}$ & $\begin{array}{l}2.29 \\
2.11\end{array}$ & $\begin{array}{l}0.37 \\
0.33\end{array}$ & $\begin{array}{r}9.04 \\
12.36\end{array}$ \\
\hline & & 22 & 105 & 27 & & & 114 & 21 & 31 & & 8. & 34 & & & & & & & & & & & 11.77 \\
\hline & $\begin{array}{l}571.7 \\
5006\end{array}$ & 20 & 108 & 24 & & & 94 & 16 & 28 & & 7.12 & 29 & 5. & & 5.25 & & 4.71 & & 2. & & 2.23 & 0.43 & 12.39 \\
\hline $1,2-$ & 599.6 & 161 & 109 & 20 & & & 81 & 14 & 22 & 4 & 6.01 & 23 & 4.8 & & 4.16 & 0 & 3.78 & & 1. & & 2.02 & 0.35 & 12.09 \\
\hline 37 & 630. & 21 & 11 & 28 & & & 10 & 18 & 27 & & 7. & 26 & $5 .($ & & 4.60 & & 4.29 & & 2. & & 1.95 & & 10.89 \\
\hline & & 1. & 76 & 18 & & & 9 & 1 & 24 & & 6.66 & 26 & 5. & & 4.54 & & 4.02 & & & & & & 14.71 \\
\hline & 69 & 18 & 87 & 21 & & & 10 & 16 & 21 & & 5.26 & 21 & 4. & 1. & 4.00 & & 3.65 & & 1. & & 1.68 & 0.29 & 10.54 \\
\hline 4 & 72 & 15 & 106 & 34 & & & 1 & 18 & 26 & & 7.28 & 29 & 6. & 1. & 5.78 & & & & 2. & & & 0.43 & 16.60 \\
\hline & 76 & 16 & 69 & 23 & & & 126 & 17 & 26 & & 7.14 & 28 & 5. & 1. & 5.18 & 0. & 4. & & & & & 0.40 & 15.21 \\
\hline $4,112-122$ & 807.42 & 156 & 72 & 19 & & & 119 & 14.4 & 23.4 & 48 & 6.65 & 25 & 5. & 1.30 & 4.75 & 0. & 4.30 & 0. & 2. & 0. & 2.03 & 0.31 & 10.62 \\
\hline 62 & 870.51 & 171 & 101 & 29 & & & 99 & 12.7 & 18. & 31 & 4.16 & 16. & 3.1 & 0.96 & 2.98 & 0. & 2.79 & 0. & 1. & 0. & 1.41 & 0.20 & 7.99 \\
\hline & & 20 & 14. & 28 & & & 154 & 10 & 25 & 10 & 6. & 26 & 5 & & 4. & & & & & & & & 7.23 \\
\hline & 82.1 & 17 & 13 & 3 & & & 1 & 17 & 36 & 66. & 10. & 38 & 7.4 & & 6. & 0. & 5. & 0.8 & & & & & 3 \\
\hline & 10 & 14 & 1 & 1 & & & 10 & & 27 & & & & & & & & & & & & & & \\
\hline & & 0 & & 18 & & & & & 16 & & & & & & & & & & & & & & \\
\hline $8 \gamma$ & 1 & 14 & 5 & 6 & & & 66 & & 18 & & 4 & 2 & 4.2 & & 4. & & & & & & & & \\
\hline & & 20 & 1 & 4 & 123 & 138 & & & & & & & & & & & & & & & & 1 & 4.31 \\
\hline $95 \mathrm{R}-2,140-150$ & 1182.20 & 211 & 154 & 59 & & & 146 & 10.0 & 11.5 & 20.3 & 3.12 & 13.8 & 3.13 & 0.80 & 3.21 & 0.47 & 2.62 & 0.45 & 1.28 & 0.17 & 1.18 & 0.17 & 3.56 \\
\hline $152-9$ & & & & & & & & & & & & & & & & & & & & & & & \\
\hline $1 \mathrm{H}-2,14$ & 2.95 & 259 & 206 & 45 & 109 & 169 & 130 & 25 & 18 & 40 & 4.8 & 20. & 4.3 & & 4.35 & & 3.98 & & 1. & 0. & 1.75 & 0.24 & 4.05 \\
\hline & 5.95 & 168 & 133 & 27 & & & 122 & 18.2 & 37.4 & 68. & 8.63 & 34. & 5.6 & 1. & 5.14 & 0 & 4.15 & 0 & 2. & 0. & 2.04 & 0.27 & 10.39 \\
\hline $2 \mathrm{H}-2,145-150$ & 10.95 & 200 & 77 & 28 & & & 123 & 26.4 & 27.3 & 53.8 & 7.23 & 32.9 & 6.81 & 1.81 & 6.67 & 1.02 & 6.46 & 1.04 & 3.46 & 0.46 & 3.31 & 0.59 & 8.16 \\
\hline
\end{tabular}


Table 2 (continued).

\begin{tabular}{|c|c|c|c|c|c|c|c|c|c|c|c|c|c|c|c|c|c|c|c|c|c|c|c|}
\hline $\begin{array}{l}\text { Core, section, } \\
\text { interval }(\mathrm{cm})\end{array}$ & $\begin{array}{l}\text { Depth } \\
\text { (mbsf) }\end{array}$ & $\begin{array}{c}\mathrm{V} \\
(\mathrm{ppm})\end{array}$ & $\begin{array}{c}\mathrm{Cr} \\
(\mathrm{ppm})\end{array}$ & $\begin{array}{c}\text { Co } \\
(\mathrm{ppm})\end{array}$ & $\begin{array}{c}\mathrm{Ni} \\
(\mathrm{ppm})\end{array}$ & $\begin{array}{c}\mathrm{Cu} \\
(\mathrm{ppm})\end{array}$ & $\begin{array}{c}\mathrm{Zn} \\
(\mathrm{ppm})\end{array}$ & $\begin{array}{c}\mathrm{Y} \\
(\mathrm{ppm})\end{array}$ & $\begin{array}{c}\mathrm{La} \\
(\mathrm{ppm})\end{array}$ & $\begin{array}{c}\mathrm{Ce} \\
(\mathrm{ppm})\end{array}$ & $\begin{array}{c}\mathrm{Pr} \\
(\mathrm{ppm})\end{array}$ & $\begin{array}{c}\mathrm{Nd} \\
(\mathrm{ppm})\end{array}$ & $\begin{array}{l}\mathrm{Sm} \\
(\mathrm{ppm})\end{array}$ & $\begin{array}{c}\mathrm{Eu} \\
(\mathrm{ppm})\end{array}$ & $\begin{array}{c}\mathrm{Gd} \\
(\mathrm{ppm})\end{array}$ & $\begin{array}{c}\mathrm{Tb} \\
(\mathrm{ppm})\end{array}$ & $\begin{array}{c}\text { Dy } \\
(\mathrm{ppm})\end{array}$ & $\begin{array}{c}\mathrm{Ho} \\
(\mathrm{ppm})\end{array}$ & $\begin{array}{c}\text { Er } \\
(\mathrm{ppm})\end{array}$ & $\begin{array}{c}\mathrm{Tm} \\
(\mathrm{ppm})\end{array}$ & $\begin{array}{c}\mathrm{Yb} \\
(\mathrm{ppm})\end{array}$ & $\begin{array}{c}\mathrm{Lu} \\
(\mathrm{ppm})\end{array}$ & $\begin{array}{c}\mathrm{Pb} \\
(\mathrm{ppm})\end{array}$ \\
\hline $2 \mathrm{H}-4,145-150$ & 13.95 & 217 & 87 & 30 & \multirow{16}{*}{58} & \multirow{12}{*}{142} & 108 & 22.5 & 22.1 & 42.4 & 5.90 & 25.0 & 5.38 & 1.48 & 5.06 & 0.80 & 5.04 & 0.90 & 2.52 & 0.32 & 2.75 & 0.36 & 7.43 \\
\hline $3 \mathrm{H}-2,145-150$ & 20.45 & 158 & 88 & 27 & & & 101 & 19.3 & 32.7 & 61.6 & 8.15 & 32.5 & 6.14 & 1.71 & 5.38 & 0.86 & 5.36 & 0.81 & 2.64 & 0.44 & 2.41 & 0.37 & 11.38 \\
\hline $3 \mathrm{H}-4,145-150$ & 23.45 & 196 & 76 & 30 & & & 104 & 23.1 & 22.8 & 45.7 & 6.62 & 29.3 & 5.99 & 1.83 & 5.98 & 0.99 & 6.41 & 1.09 & 3.13 & 0.43 & 3.16 & 0.41 & 8.88 \\
\hline $4 \mathrm{H}-2,145-150$ & 29.95 & 188 & 80 & 31 & & & 109 & 24.7 & 24.1 & 47.5 & 6.87 & 28.6 & 6.69 & 1.97 & 6.42 & 0.99 & 6.55 & 1.12 & 3.15 & 0.40 & 3.10 & 0.50 & 7.62 \\
\hline $4 \mathrm{H}-4,145-150$ & 32.95 & 205 & 97 & 30 & & & 115 & 22.0 & 23.2 & 43.6 & 6.05 & 24.8 & 5.08 & 1.38 & 4.98 & 0.72 & 4.66 & 0.69 & 2.35 & 0.32 & 2.43 & 0.36 & 9.32 \\
\hline $5 \mathrm{H}-2,145-150$ & 39.45 & 219 & 100 & 33 & & & 102 & 22.2 & 18.1 & 36.4 & 5.23 & 23.6 & 5.37 & 1.65 & 5.83 & 0.83 & 6.07 & 0.97 & 2.98 & 0.43 & 2.95 & 0.49 & 5.59 \\
\hline $5 \mathrm{H}-4,145-150$ & 42.45 & 244 & 103 & 37 & & & 119 & 24.6 & $\begin{array}{l}10.1 \\
18.1\end{array}$ & 36.5 & 5.61 & 24.6 & 5.59 & 1.72 & 5.60 & 0.90 & 5.99 & 1.06 & 3.14 & 0.42 & 2.82 & 0.47 & 5.39 \\
\hline $6 \mathrm{H}-3,145-150$ & 50.45 & 251 & 117 & 37 & & & 102 & 21.6 & 17.7 & 33.8 & 4.94 & 21.8 & 4.89 & 1.50 & 5.10 & 0.69 & 5.24 & 0.77 & 2.46 & 0.37 & 2.46 & 0.35 & 5.19 \\
\hline $7 \mathrm{H}-3,145-150$ & 59.95 & 132 & 76 & 16 & & & 101 & 19.9 & 35.4 & 66.6 & 8.80 & 33.9 & 6.27 & 1.39 & 5.10 & 0.72 & 4.78 & 0.76 & 2.31 & 0.39 & 2.25 & 0.35 & 19.45 \\
\hline $8 \mathrm{H}-3,145-150$ & 69.45 & 271 & 96 & 41 & & & 135 & 27.7 & 20.7 & 43.5 & 5.51 & 22.3 & 4.88 & 1.42 & 5.22 & 0.75 & 4.48 & 0.67 & 2.07 & 0.28 & 1.83 & 0.31 & 5.19 \\
\hline $9 \mathrm{H}-3,145-150$ & 78.95 & 197 & 83 & 28 & & & 112 & 22.2 & 26. & 52.3 & 6.58 & 28.8 & 5.7 & 1.48 & 5.22 & 0.78 & 5.0 & 0.89 & 2.45 & 0.38 & 2.38 & 0.39 & 10.08 \\
\hline $10 \mathrm{H}-3,145-150$ & 88.45 & 268 & 124 & 39 & & & 113 & 22.9 & 18.6 & 36.4 & 5.08 & 22.6 & 5.05 & 1.57 & 4.87 & 0.83 & 5.25 & 0.88 & 2.53 & 0.42 & 2.61 & 0.41 & 6.01 \\
\hline \multicolumn{23}{|l|}{ 152-919B- } & \\
\hline $3 \mathrm{H}-3,145-150$ & 94.45 & 192 & 79 & 31 & & & 110 & 21. & 22.7 & 45.6 & 5.97 & 27.2. & 5.6 & 1.5 & 5.60 & 0.80 & 5.55 & 0.88 & 2.90 & 0.45 & 2.72 & 0.46 & 9.17 \\
\hline $4 \mathrm{H}-3,145-150$ & 103.95 & 217 & 91 & 32 & & & 116 & 25. & 26.3 & 51.7 & 7.12 & 30. & 6.15 & 1.67 & 5.97 & 0.87 & 5.99 & 0.94 & 3.05 & 0.44 & 2.73 & 0.41 & 10.27 \\
\hline $7 \mathrm{H}-3,145-150$ & 132.45 & 143 & 107 & 24 & & & 127 & 17.9 & 43.3 & 77.3 & 10.10 & 39.5 & 6.47 & 1.59 & 5.31 & 0.76 & 4.50 & 0.72 & 2.35 & 0.32 & 2.29 & 0.35 & 12.08 \\
\hline
\end{tabular}

Note: For Site 918, data are arranged in order of depth (mbsf), not according to hole. 